Matrix metalloproteinase-13 (MMP-13) has a potential role in tumour invasion and metastasis. However, its relevance to the prognosis of human breast cancer is poorly understood. The aim of this study is to investigate the expression patterns of MMP-13 protein and to determine its prognostic value in breast cancer, and to define its relation to the clinicopathological features. Immunohistochemistry analysis of MMP-13 was performed on formalin-fixed, paraffin-embedded sections of cancerous breast tissue $(n=76)$ and normal breast tissue $(n=20)$, all of which had clinicopathological information available. Based on the principle of immunoreactivity, the detection of MMP-13 on breast tissue was conducted using monoclonal antibodies against MMP-13. A semi-quantitative scoring system was used to assess the presence of, as well as the cellular localisation of MMP-13. MMP-13 expression was significantly greater in the cancerous breast tissues in comparison to those of normal breast tissues. In addition, high levels of MMP-13 expression were also found to be related to the positive detection of breast cancer cells in lymph nodes-amongst breast cancer patients. The results of this study showed that MMP-13 was frequently present in breast tumours, especially when tumours were accompanied by positive breast cancer cell detection in lymph nodes. This suggests that MMP-13 plays a potentially significant role in breast cancer invasion and metastasis.

Key words: MMP-13, breast cancer, metastasis, Hatyai, Thailand.

Contemp Oncol (Pozn); 2016; 20 (3): 225-228 DOI: $10.5114 /$ wo.2016.61565

\section{Differential expression of matrix metalloproteinase-13 in association with invasion of breast cancer}

\author{
Manas Kotepui ${ }^{1}$, Chuchard Punsawad ${ }^{2}$, Chaowanee Chupeerach ${ }^{3}$, \\ Apiram Songsri ${ }^{4}$, Lek Charoenkijkajorn ${ }^{4}$, Songsak Petmitr ${ }^{5}$
}

\begin{abstract}
${ }^{1}$ Medical Technology Program, School of Allied Health Sciences and Public Health, Walailak University, Nakhon Si Thammarat, Thailand

${ }^{2}$ School of Medicine, Walailak University, Nakhon Si Thammarat, Thailand

${ }^{3}$ Institute of Nutrition, Mahidol University, Nakhon Pathom, Thailand

${ }^{4}$ Department of Pathology, Hatyai Hospital, Songkhla, Thailand

${ }^{5}$ Department of Molecular Tropical Medicine and Genetics, Faculty of Tropical Medicine, Mahidol University, Bangkok, Thailand
\end{abstract}

\section{Introduction}

Breast cancer is the most common type of cancer amongst women worldwide, including Thailand, and is responsible for a high number of fatalities on a yearly basis [1]. Early diagnosis of breast cancer can provide patients with a wider range of therapeutic options as well as a higher success rate of therapy with lower mortality rate, which is the key to higher survival rates [2]. Clinical parameters such as the size of the tumour, the histological grade, and regional lymph node involvement are generally used for predicting the prognosis of breast cancer. Previous studies have also reported that Oestrogen receptor (ER)/progesterone receptor (PR) and Her-2/neu are useful markers for breast cancer diagnosis $[3,4]$.

Epithelial basement membrane and extracellular matrix (ECM) are composed of a tough network of fibrillar ECM proteins. Tumour invasion and metastasis involve the degradation of these components of the ECM and require the actions of proteolytic enzymes, such as matrix metalloproteinases (MMPs) [5, 6]. Human collagenase-3 (MMP-13) was one such MMP first identified in human breast carcinomas [7]. MMP-13 is synthesised as a latent pro-enzyme and undergoes activation by cleavage of the N-terminal peptide [8]. Expression of MMP-13 has been detected in various types of cancers, such as papillary thyroid carcinoma [9] and colorectal cancer [10], as well as breast cancer $[11,12]$. Recent studies have further suggested that MMP-13 may play a central role in the extracellular MMP activation cascade, resulting in degradation of the ECM network $[13,14]$.

The goal of this study is to evaluate the expression and prognostic value of MMP-13 and its tissue distribution pattern in human breast cancer and normal (non-cancerous) tissues. The increase of MMP-13 expression in breast cancer might independently be a predictor of poor prognosis of the cancer.

\section{Material and methods}

Human subjects and tissue specimens

Invasive ductal breast cancer tissues diagnosed and surgically treated between January 2009 and December 2012 at the Department of Pathology, Hatyai Hospital, Songkhla Province, Thailand were collected. Normal breast tissues diagnosed by a pathologist were obtained from the same department. Patients had not received prior radiotherapy or neoadjuvant therapies before the recruitment of the tissues. The available information of individual patients included age at diagnosis, type of tissue, grade of tumour, and 
Table 1. Patient demographic characteristics

\begin{tabular}{ll} 
Characteristic & Number (\%) \\
Age; Mean (SD) & $50.3(10.9)$ \\
Type of tissues & $76(79.2)$ \\
Tumour & $20(20.8)$ \\
Normal & \\
Lymph node status & $44(57.9)$ \\
Positive & $32(42.1)$ \\
Negative & $17(22.4)$ \\
Differentiation of tumour & $55(72.4)$ \\
Well & $4(5.3)$ \\
Moderate & \\
\hline
\end{tabular}

regional lymph node status. Grading standard was then used to assign scores of histological grades of breast cancer to the samples collected. Grade I refers to well-differentiated tumours, grade II refers to moderately differentiated tumours, and grade III refers to poorly differentiated tumours. This study included a total of 76 cases of invasive ductal breast cancer and 20 normal breast tissues.

This study was performed under a protocol approved by the Ethics Committee of Hatyai Hospital and the Ethical Clearance Committee on Human Rights Related to Research Involving Human Subjects of Walailak University. This research was performed at Walailak University and was conducted for two years: from August 2013 to July 2014.

\section{Immunohistochemistry}

Briefly, consecutive 5- $\mu$ m-thick sections were cut from the recipient blocks and placed on a poly-L-lysine-coated slide for immunochemistry $(\mathrm{IHC})$ analysis. IHC was performed using the method described previously [15]. The MMP-13 specific antibodies, with dilution factor of $1: 200$ (Abcam, USA), were diluted using blocking buffer

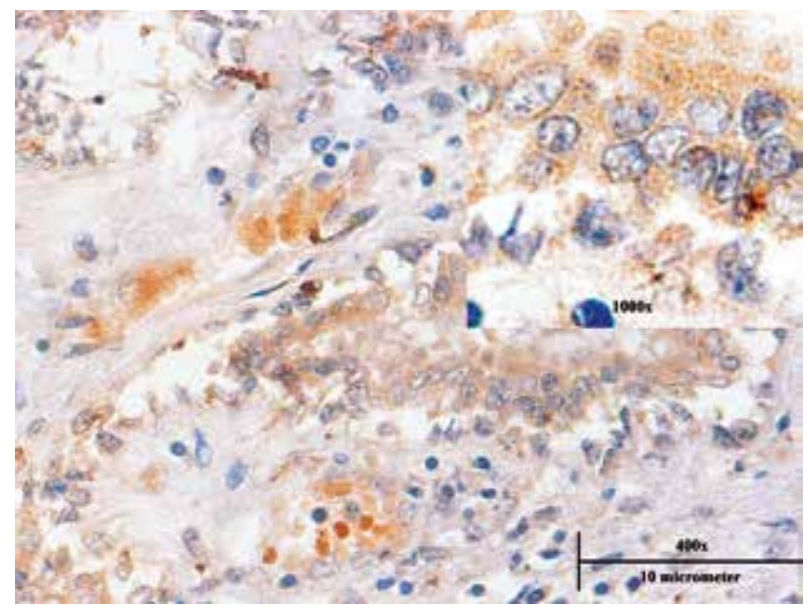

Fig. 1. Immunohistochemical localisation of matrix metalloprotein ase 13 (MMP-13) in breast cancer
(PBS with normal serum). Blocking buffer was used in place of the primary antibodies in all negative controls of IHC.

\section{Semi-quantitative measurement of immunostaining}

Immunohistochemistry was scored independently using a semi-quantitative scoring system, described below. Discordant scores were re-evaluated by the investigators and the consensus scores were used for further analyses. Both the intensity and the percentage of positive cells from IHC were assessed [15]. The intensity of the immunostaining was classified into four groups: no brown particle staining (0), light brown particle (1), moderate brown particle (2), and dark brown particle staining in cytoplasm (3). The percentage of positive cells, like the extent of immunostaining, was quantified under a microscope and then classified into four groups: < $25 \%$ positive cells (1), $25 \%$ to $50 \%$ positive cells (2), $51 \%$ to $75 \%$ positive cells (3), and $>75 \%$ positive cells (4). The staining index (SI), the product of the intensity, and the percentage of positive staining were used to define high $(\mathrm{SI} \geq 6)$ or low $(\mathrm{SI}<6)$ expressions of MMP-13.

\section{Statistical analysis}

Statistical analyses were performed using the SPSS software package 11.0 (SPSS, Inc., Chicago, IL, USA). The correlations between MMP-13 expression and clinicopathological variables were analysed using the Pearson Chi-square test. All of the statistical tests were two-sided, and a $p$-value of less than 0.05 was considered statistically significant.

\section{Results}

This study included a total of 76 cases of invasive ductal breast cancer and 20 normal breast tissues. From the tumour cases, 17 cases (22.4\%) were Grade I tumours, 55 (72.4\%) cases were Grade II tumours, and 4 cases (5.3\%) were Grade III tumours. A total of 44 cases (57.9\%) were lymph node-positive, while 32 cases (42.1\%) were lymph node-negative (Table 1).

To examine the expression of MMP-13 at a cellular level, immunohistochemistry was performed to analyse the presence of MMP-13 via the immunoreactivity of MMP-13 to MMP-13 specific antibodies. The intracellular staining allowed for the detection of MMP-13 localised to the cytoplasm of tumour cells (Fig. 1). The proportion of immunopositive tumour cells in each group is shown in Fig. 2, whereas the intensity of immunostaining for tumours is shown in Fig. 3.

To test the potential value of MMP-13 as a breast cancer biomarker, Pearson Chi-square analysis was performed to evaluate the correlation of MMP-13 expression with clinical and histopathological features (age at diagnosis, type of tissues, tumour grade, and lymph node status) that were further stratified (Table 2). A p-value of less than 0.05 was considered statistically significant. The tumour staining was semi-quantitatively scored by the SI scales and assigned into high and low categories. No correlation between age at diagnosis and expression of MMP-13 was 


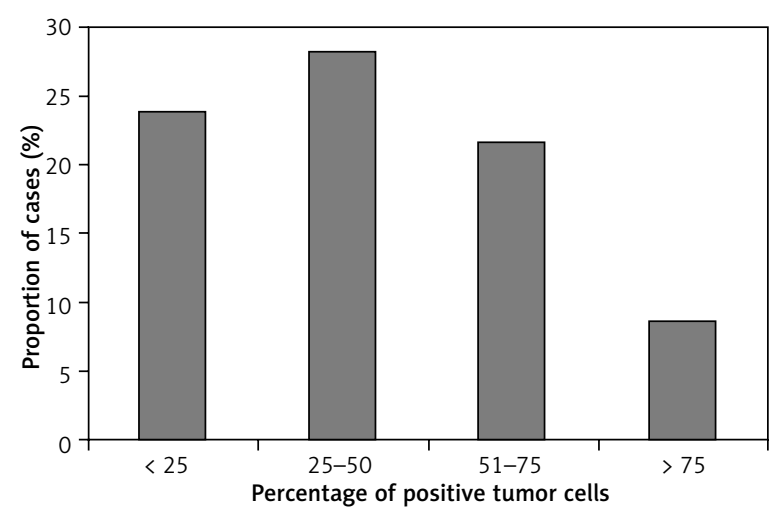

Fig. 2. The frequency distribution of the percentage of matrix metalloproteinase 13-positive tumour cells

found ( $p=0.472$, odds ratio $=1.41,95 \% \mathrm{Cl}$ : 0.55-3.63). High levels of MMP-13 expression were correlated with the type of tissues examined $(p=0.034$, odds ratio $=34.5,95 \% \mathrm{Cl}$ : 4.37-271.9). High levels of MMP-13 expression were also correlated with lymph node status ( $p=0.034$, odds ratio $=$ $0.34,95 \% \mathrm{Cl}$ : 0.12-0.94). Among lymph node-positive cases and lymph node-negative cases, twenty cases (74\%) and seven cases (26\%) were SI index less than 6 , respectively.

\section{Discussion}

Tumour invasion and metastasis are the main causes of breast cancer-related deaths. ECM modification and angiogenesis are essential processes in facilitating cancer cell invasion and metastasis. The results of this study showed that the MMP-13 protein expression level in breast cancer tissues was significantly higher than that in normal breast tissues, which suggested that MMP-13 expression could be upregulated in the tumorigenesis and progression of human breast cancer. MMP-13 staining was localised to the cytoplasm of tumour cells. This was consistent with studies of other tumours, including colorectal cancer [16] and papillary thyroid carcinoma, [9] which found that MMP-13 expression was primarily present in cytoplasm of tumour cells. Moreover, MMP-13 expression was detected in over $81 \%$ of tumour cases examined. This indicated that the MMP-13 was expressed predominantly in tumour cells and were only occasionally weakly expressed in normal cells. The frequency of MMP-13 expression in breast cancer was similar to the immunohistochemical studies of a previous study [11]. The increase in MMP-13 may represent MMP-13 upregulation, with high amounts of MMP-13 being required for the activation of other MMPs. Furthermore, it is rational that tumours have far higher amounts of MMP-13 activity because this molecule catalyses the breakdown of ECM required for its invasion and further metastasis. On the other hand, normal breast cells do not require MMP-13 mediated ECM breakdown.

Regional lymph node status is the most important prognostic factor in breast cancer. Prognosis worsens with the increase in the number of tumour-positive lymph nodes [17]. This study suggests that MMP-13 probably plays a role in promoting tumour invasion and metastasis or promotes

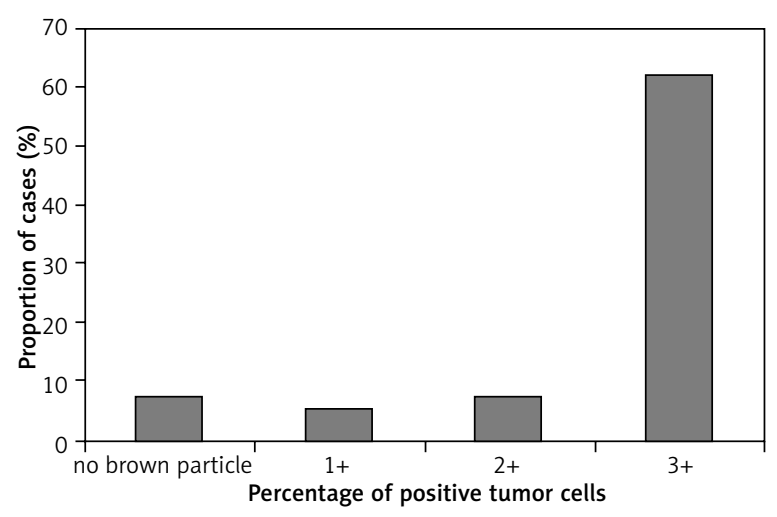

Fig. 3. The frequency distribution for the intensity of matrix metalloproteinase 13 (MMP-13) immunohistochemical staining in breast cancer

the catalysis of ECM breakdown, both in patients with and without positive lymph node invasion. Since MMP-13 is a metalloproteinase, it may act in a similar manner as MMP-2 and MMP-9. Both MMP-2 and MMP-9 have been extensively studied as biomarkers and therapeutic targets in breast cancer $[18,19]$. Regardless of the mechanism, these findings suggest a role for MMP-13 in breast cancer progression.

Conclusively, the results of this study show that MMP13 is frequently present in breast cancer, especially in breast cancer patients with positive lymph node invasion, which might suggest its role in breast cancer progression and metastasis. Thus the prognostic value of abnormal MMP-13 expression in cancer tissues and the detailed mechanism of the up-regulation of MMP-13 expression in carcinogenesis could further be investigated to clarify its role in breast cancer. In addition, further study, with higher sample size and a method with higher validity, is needed to confirm the mechanism of MMP-13 expression.

Table 2. Clinicopathological characteristics of the patients analysed by MMP-13 expression

\begin{tabular}{|c|c|c|c|}
\hline \multirow{2}{*}{$\begin{array}{l}\text { Clinicopathological } \\
\text { characteristics }\end{array}$} & \multicolumn{2}{|c|}{ Staining index (SI) } & \multirow[t]{2}{*}{$p$-value* } \\
\hline & $\mathrm{SI}<6$ & $\mathrm{SI} \geq 6$ & \\
\hline \multicolumn{4}{|l|}{ Age (years) } \\
\hline$<50$ & $12(31.6)$ & $26(68.4)$ & \\
\hline$\geq 50$ & $15(39.5)$ & $23(60.5)$ & 0.472 \\
\hline \multicolumn{4}{|l|}{ Type of tissues } \\
\hline Tumour & $49(64.5)$ & $27(35.5)$ & \\
\hline Normal & $1(5)$ & $19(95)$ & $<0.001^{\star *}$ \\
\hline \multicolumn{4}{|l|}{ Lymph node status } \\
\hline Positive, $n$ (\%) & $20(45.5)$ & $24(54.5)$ & \\
\hline Negative, $n(\%)$ & $7(21.9)$ & $25(78.1)$ & $<0.034^{* *}$ \\
\hline \multicolumn{4}{|c|}{ Differentiation of tumour } \\
\hline Well & $7(41.2)$ & $10(58.8)$ & \\
\hline Moderate & $17(30.9)$ & $38(69.1)$ & \\
\hline Poor & $3(75)$ & $1(25)$ & 0.176 \\
\hline
\end{tabular}

${ }^{*} p$-value by Pearson $\chi^{2}$ test, ${ }^{* *}$ Statistical significant 
The authors would like to thank the staff from the Department of Pathology, Hatyai Hospital, Songkhla, Thailand for their support and assistance, and for giving us the opportunity to do this project. This study was supported by funding from Walailak University (WU56315).

The authors declare no conflict of interest.

\section{References}

1. Ferlay J SI, Ervik M, Dikshit R, Eser S, Mathers C, Rebelo M, Parkin DM, Forman D, Bray, F. GLOBOCAN 2012 v1.0, Cancer Incidence and Mortality Worldwide: IARC CancerBase No. 11. In Edition Lyon, France: International Agency for Research on Cancer 2013.

2. Chang HJ, Yang MJ, Yang YH, et al. MMP13 is potentially a new tumor marker for breast cancer diagnosis. Oncol Rep 2009; 22: 1119-27.

3. Tavassoéli FA, Devilee, P. Pathology and Genetics Tumours of the Breast and Female Genital Organs. WHO Press, Lyon 2003.

4. Choi DH, Shin DB, Lee $M H$, et al. A comparison of five immunohistochemical biomarkers and HER-2/neu gene amplification by fluorescence in situ hybridization in white and Korean patients with early-onset breast carcinoma. Cancer 2003; 98: 1587-95.

5. Katiyar SK. Matrix metalloproteinases in cancer metastasis: molecular targets for prostate cancer prevention by green tea polyphenols and grape seed proanthocyanidins. Endocr Metab Immune Disord Drug Targets 2006; 6: 17-24.

6. Price JT, Thompson EW. Mechanisms of tumour invasion and metastasis: emerging targets for therapy. Expert Opin Ther Targets 2002; 6: 217-33.

7. Freije JM, Diez-Itza I, Balbin M, et al. Molecular cloning and expression of collagenase-3, a novel human matrix metalloproteinase produced by breast carcinomas. J Biol Chem 1994; 269: 16766-73.

8. Knauper V, Lopez-Otin C, Smith B, et al. Biochemical characterization of human collagenase-3. J Biol Chem 1996; 271: 1544-50.

9. Wang JR, Li XH, Gao XJ, et al. Expression of MMP-13 is associated with invasion and metastasis of papillary thyroid carcinoma. Eur Rev Med Pharmacol Sci 2013; 17: 427-35.

10. Yang B, Gao J, Rao Z, Shen Q. Clinicopathological significance and prognostic value of MMP-13 expression in colorectal cancer. Scand J Clin Lab Invest 2012; 72: 501-5.

11. Zhang B, Cao X, Liu Y et al. Tumor-derived matrix metalloproteinase-13 (MMP-13) correlates with poor prognoses of invasive breast cancer. BMC Cancer 2008; 8: 83.

12. Kotepui M, Thawornkuno C, Chavalitshewinkoon-Petmitr P, et al. Quantitative real-time RT-PCR of ITGA7, SVEP1, TNS1, LPHN3, SEMA3G, KLB and MMP13 mRNA expression in breast cancer. Asian Pac J Cancer Prev 2012; 13: 5879-82.

13. Ala-aho R, Kahari VM. Collagenases in cancer. Biochimie 2005; 87: 273-86.

14. Leeman MF, Curran S, Murray GI. The structure, regulation, and function of human matrix metalloproteinase-13. Crit Rev Biochem Mol Biol 2002; 37: 149-66.

15. Cao W, Zhang B, Liu Y, et al. High-level SLP-2 expression and HER$2 /$ neu protein expression are associated with decreased breast cancer patient survival. Am J Clin Pathol 2007; 128: 430-6.

16. Leeman MF, McKay JA, Murray GI. Matrix metalloproteinase 13 activity is associated with poor prognosis in colorectal cancer. J Clin Pathol 2002; 55: 758-62.

17. Wu CW, Hsieh MC, Lo SS, et al. Relation of number of positive lymph nodes to the prognosis of patients with primary gastric adenocarcinoma. Gut 1996; 38: 525-7.

18. Jinga DC, Blidaru A, Condrea I, et al. MMP-9 and MMP-2 gelatinases and TIMP-1 and TIMP-2 inhibitors in breast cancer: correlations with prognostic factors. J Cell Mol Med 2006; 10: 499-510.

19. Li HC, Cao DC, Liu Y, et al. Prognostic value of matrix metalloproteinases (MMP-2 and MMP-9) in patients with lymph node-negative breast carcinoma. Breast Cancer Res Treat 2004; 88: 75-85.

\section{Address for correspondence}

\section{Manas Kotepui}

Medical Technology Program

School of Allied Health Sciences and Public Health

Walailak University

Nakhon Si Thammarat, Thailand

tel. 66860390260

fax 6675672106

e-mail: manas.ko@wu.ac.th

Submitted: 2.12 .2014

Accepted: $\quad 15.10 .2015$ 